\title{
An improved photometric stereo through distance estimation and light vector optimization from diffused maxima region
}

\author{
Jahanzeb Ahmad ${ }^{1}$, Jiuai Sun, Lyndon Smith, Melvyn Smith \\ Centre for Machine Vision, Bristol Robotics Laboratory, University of the West of \\ England, Bristol, UK
}

\begin{abstract}
Although photometric stereo offers an attractive technique for acquiring 3D data using low-cost equipment, inherent limitations in the methodology have served to limit its practical application, particularly in measurement or metrology tasks. Here we address this issue. Traditional Photometric Stereo assumes that lighting directions at every pixel are the same, which is not usually the case in real applications, and especially where the size of object being observed is comparable to the working distance. Such imperfections of the illumination may make the subsequent reconstruction procedures used to obtain the 3D shape of the scene prone to low frequency geometric distortion and systematic error (bias). Also, the 3D reconstruction of the object results in a geometric shape with an unknown scale. To overcome these problems a novel method of estimating the distance of the object from the camera is developed, which employs photometric stereo images without using
\end{abstract}

Email addresses: jahanzeb.ahmad@uwe.ac.uk (Jahanzeb Ahmad), jiuai2.sun@uwe.ac.uk (Jiuai Sun), Iyndon.smith@uwe.ac.uk (Lyndon Smith), melvyn.smith@uwe.ac.uk (Melvyn Smith)

${ }^{1}$ Corresponding Author 
other additional imaging modality. The method firstly identifies Lambertian diffused maxima region to calculate the object distance from the camera, from which the corrected per-pixel light vector is able to be derived and the absolute dimensions of the object can be subsequently estimated. We also propose a new calibration process to allow a dynamic(as an object moves in the field of view) calculation of light vectors for each pixel with little additional computation cost. Experiments performed on synthetic as well as real data demonstrates that the proposed approach offers improved performance, achieving a reduction in the estimated surface normal error of up to $45 \%$ as well as mean height error of reconstructed surface of up to $6 \mathrm{~mm}$. In addition, when compared to traditional photometric stereo, the proposed method reduces the mean angular and height error so that it is low, constant and independent of the position of the object placement within a normal working range.

Keywords: Photometric Stereo, Light Vector Calculation, Distance Estimation.

\section{Introduction}

2 Traditional Photometric Stereo (PS) is used to recover the surface shape 3 of an object or scene by using several images taken from the same view 4 point but under different controlled lighting conditions [1, 2]. It was initially 5 introduced by Woodham in 1980 [3]. PS has been extensively used in many 6 applications especially for estimating high density local surface normals in 7 the fields of computer vision and computer graphics. It has been used for

8 3D modelling [4], facial expression capturing [5, 6]. It has also been used for 
medical applications $[7,8]$ and in face recognition security systems [9]. Most of these applications require high accuracy reconstructed surfaces. So it is critical to estimate high accuracy surface normals in order to get accurate subsequent surface reconstruction from their integration. As we will show in the following experiment setup, 2-3 degree error in surface normal can produce up to $6 \mathrm{~mm}$ error in the height of reconstructed surface.

Current state-of-the-art systems normally assume that light sources are at an infinite distance from the scene so that a homogeneous and parallel incident light condition can be formed; and then the PS problem becomes solvable through a group of linear equations. In reality it is not always possible to produce parallel(collimated) incident light, especially when the object size is comparable in magnitude to the light separation and or the distance of object from light source is relatively small. Any underestimation or misalignment of the illumination may produce some error during recovery of the surface normal. For example, a $1 \%$ uncertainty in the intensity estimation will cause a 0.5-3.5 degree deviation in the calculated surface normal for a typical three-light source photometric stereo setup [10]. Uncertainty in the calibration process can also lead to systemic errors when recovering surface normals and in the $3 \mathrm{D}$ recovered surface $[11,12]$.

Furthermore PS gives no information concerning the absolute distance of the object from the camera. Other imaging modalities are normally required for obtaining such range data, for example laser triangulation or stereo vision techniques have been combined with the PS approach [13-17]. A dense (perpixel) surface reconstruction of a smooth and texture-less object proves to be a challenging task for many range detection imaging approaches, since they 
can only provide sparse surface data. In order to recover the range data at pixel resolution, we may alternatively make use of some information about the object surface itself such as convexity and smoothness.

In this paper we present a novel method to allow us to calculate the distance of an object based on the same photometric stereo imaging setup, i.e. one camera and four lights, without a requirement for any additional hardware, but with little extra computation processing cost. The object's distance from the camera is estimated by finding small patches on the object surface whose normal is pointing towards light source. This small patch is also called the diffused maxima region (DMR) and has been recently [18] used for solving the problem of the generalized bas-relief ambiguity (GBR) [19]. The estimated distance is then used to calculate the light vectors at every image pixel, thereby minimizing the error associated with the assumption of a collimated light source. This approach enables the photometric stereo method to effectively work with real light sources, on Lambertian surfaces that have at least one patch with normal vectors pointing directly towards the light source, in reality this is a reasonable assumption.

To the best of our knowledge we are the first to use the DMR in this way, i.e. to enhance the PS method by reducing the well know problem of distortion in the recovered 3D surface by improving the light vector direction estimation and adding range data by using the convexity and smoothness of real objects and without using other additional imaging modalities. Paper is organized as following, in next section we will discuss related work after that photometric stereo technique is discussed. In section 4 proposed method is discussed followed by experiments and results in section 5. Finally in section 
6 paper is concluded.

\section{Related Work}

The common low cost approach to produce collimated light is to use convex lenses or concave mirrors; but even in these cases, only a narrow parallel light beam with similar physical size to that of the lens or mirror can be obtained. To produce a collimated light source for a larger scene area, a possible solution is to develop a custom optical system with an array of specially aligned individual light units. Unfortunately this results in a high hardware and setup cost [20]. Another practical solution is to set the light sources far away from the object[21], so that the light can be approximated as a distant radiation point source. This strategy may help to provide evenly distributed radiance across the object surface, but it sacrifices the majority of the illumination intensity, and correspondingly decreases the signal/noise ratio of the whole system. In addition, such a distant lighting setup usually means a large impractical working space is required. So this approach is only suitable for those light sources able to produce high levels of energy and those applications where a large redundant space is available. In terms of the availability and flexibility of current commercial illumination, the distant illumination solution is often not an optimal choice.

A nearby light source model has been considered as an alternative by Kim [22] and Iwahori [23] to reduce the photometric stereo problem to find a local depth solution using a single non-linear equation. By distributed the light sources symmetrically in a plane perpendicular to camera optical axis, they were able to get a unique solution of non-linear equations. However, selection 
of initial values for the optimisation process and limitations in the speed for solving non-linear equation are the main problems with this method.

A moving point light source based solution has been proposed by Clark [24] termed "Active Photometric Stereo". By moving a point light along a known path close to the object surface a linear solution can be formulated to solve the photometric stereo problem. However, the range of motion of light must be closely controlled in order to guarantee the efficiency of the solution.

Kozera and Noakes introduced an iterative 2D Leap-Frog algorithm able to solve the noisy and non-distant illumination issue for three light-source photometric stereo [25]. Because distributed illuminators are commercially available, Smith et al. approximated two symmetrically distributed nearby point sources as one virtual distant point light source for their dynamic photometric stereo method [26]. Unfortunately, none of these methods lend themselves to a generalized approach.

Varnavas et al. [27] implemented parallel CUDA based architecture and computed light vectors at each pixel by manually placing shiny sphere at the four corners of the field of view and assuming a flat plane at that distance, so that a changing light direction was taken into account. However in practice the whole surface of the object is not flat and is not necessarily at the same distance from the light source, especially when the size of the object is comparable to the distance of the light source.

\section{Photometric Stereo}

According to the Lambertian reflectance model the intensity I of light reflected from an object's surface is dependent on the surface albedo $\rho$ and 
122

the cosine of the angle of the incident light as described in Equation 1. The cosine of the incident angle can also be referred as dot product of the unit vector of the surface normal $\vec{N}$ and the unit vector of light source direction $\vec{L}$, as shown in Equation 2 .

$$
\begin{aligned}
& I=\rho \cos \left(\phi_{i}\right) \\
& I=\rho(\vec{L} \cdot \vec{N})
\end{aligned}
$$

When more than two images (four images are used in the following work) from same view point are available under different lighting conditions, we have a linear set of Equation 1 and 2 and this can be represented in vector form as shown in Equation 3.

$$
\vec{I}(x, y)=\rho(x, y)[L] \vec{N}(x, y)
$$

${ }_{5} \quad \vec{I}$ is the vector formed by the four pixels $\left(\left(I^{1}(x, y), I^{2}(x, y), I^{3}(x, y)\right.\right.$, $\left.I^{4}(x, y)\right)^{T}$ from four images, $[L]$ is the matrix composed by the light vectors $\left(\overrightarrow{L^{1}} ; \overrightarrow{L^{2}} ; \overrightarrow{L^{3}} ; \overrightarrow{L^{4}}\right)$. Where, $1,2,3$ and 4 is the number with respect to the individual light source direction. $[L]$ is not a square and so not invertible, but the least square method can be used to compute Pseudo-Inverse and local surface gradients $p(x, y)$ and $q(x, y)$, and the local surface normal $N(x, y)$ can be calculated from the Pseudo-Inverse using Equations 4,5 and 6 where $\vec{M}(x, y)=\left(m_{1}(x, y), m_{2}(x, y), m_{3}(x, y)\right)$.

$$
\vec{M}(x, y)=\rho(x, y) N(x, y)=\left([L]^{T}[L]\right)^{-1}[L]^{T} \vec{I}(x, y)
$$




$$
\begin{gathered}
p(x, y)=\frac{m_{1}(x, y)}{m_{3}(x, y)}, q(x, y)=\frac{m_{2}(x, y)}{m_{3}(x, y)} \\
N(x, y)=\frac{p(x, y), q(x, y), 1}{\sqrt{p(x, y)^{2}+q(x, y)^{2}+1}} \\
\rho(x, y)=\sqrt{m_{1}^{2}(x, y)+m_{2}^{2}(x, y)+m_{3}^{2}(x, y)}
\end{gathered}
$$

\section{Proposed Method}

By estimating the distance of the object from the camera we can improve the accuracy of the surface normals by calculating the light vector of every pixel based on its distance from the camera and light source. The proposed method is summarised in Pseudo code in Table 1. It is divided into three parts: "Light source position estimation", "Object distance estimation" and "Per pixel light direction calculation". Light source position estimation is required only once during the rig calibration process.

\subsection{Light source position estimation}

The general assumption that the light vector is the same at every point (pixel) is mostly not true in practice, so subsequently we use triangulation and the intersection of at least two light vectors (calculated at different positions) to determine the true position of a light in a world coordinate system at the optical centre as shown in Figure 1. A specular sphere is used to calculate the light vectors at several (we take two as example) different locations in the imaging area. The intersection of these light vectors is taken as the position of the light in the real world coordinate system. The position of 
Table 1: Pseudo code of proposed method.

\section{Light Source Position Estimation.}

1.1. Place a specular sphere in Field of view.

1.2. Calculate Light vector using equation 8 .

1.3. Calculate position of highlight point in world coordinates using equation 19

1.4. Repeat steps 1.1 to 1.3 by placing the sphere in another location.

1.5. Once two light vectors and two highlight positions for same light has been calculated using the above steps, the position of light can be calculated using equation 11

1.6. Repeat steps 1.1 to 1.5 for all light sources to calculate light positions.

1.7. Calculate light vectors of all lights by placing the sphere in the centre of field of view by using equation 8 . These light vectors will be called pseudo light vectors.

\section{Object distance estimation.}

2.1. Capture a sequence of images of the object.

2.2. Calculate surface normals by using pseudo light vectors and equation 6. Resultant normals are called pseudo normals.

2.3. Calculate diffused maximum region by using equation 20 .

2.4. Create a vector from centre of diffused maximum region to centre of lens as shown in Figure 3(a).

2.5. Now using origin of lens, pseudo light vector, position of light and vector created in step 2.4 we can calculate distance of object by using the same intersection equations as used in step 1.5.

2.6. Repeat steps 2.2 to 2.4 for every light source and take average of all estimated distance values for final estimated value.

3. Per pixel light direction calculation.

3.1. Draw an imaginary plan at the estimated distance.

3.2. Calculate vectors from light position to every pixel on the plane to obtain per-pixel light vectors. 
light 1 is calculated by finding the intersection point of light vectors $\overrightarrow{L_{1}^{1}}$ and $\overrightarrow{L_{2}^{1}}$ as shown in Figure 1. $\overrightarrow{L_{1}^{1}}$ is the light vector calculated at a sphere surface position $p_{1}^{1}$ by placing the sphere at one random location and $\overrightarrow{L_{2}^{1}}$ is the light vector calculated at a sphere surface position $p_{2}^{1}$ by placing the sphere at another random location in the imaging area. To calculate $\overrightarrow{L_{1}^{1}}$ and $\overrightarrow{L_{2}^{1}}$ Equation 8 is used.

$$
\vec{L}=2(\vec{n} \cdot \vec{d}) \vec{n}-\vec{d}
$$

Where $\vec{d}$ is reflection direction taken as $(0,0,1), \vec{n}$ is unit surface normal at point $p_{1}^{1}$ or $p_{2}^{1}, \vec{n}=(n x, n y, n z), n x=p x-c x, n y=p y-c y$ and $n_{z}=\sqrt{\left(r^{2}-n_{x}^{2}-n_{y}^{2}\right)},(c x, c y)$ and $(p x, p y)$ are the pixel coordinates of the sphere centre and the highlight on the sphere respectively, and $\mathrm{r}$ is the radius of sphere in the image plane.

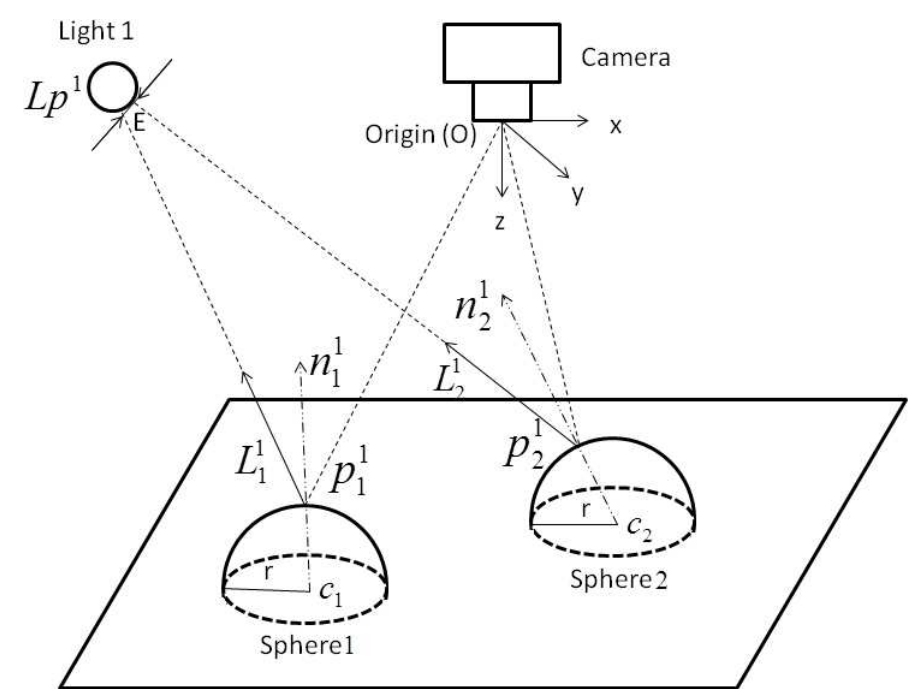

Figure 1: Calibration setup for light position calculation and initial (Pseudo) light vector calculation. 

$11[28]$

$$
\begin{gathered}
L p_{1}^{1}=p_{1}^{1}+\left(\frac{\left(\overrightarrow{L_{2}^{1}} \times\left(p_{1}^{1}-p_{2}^{1}\right)\right) \cdot\left(\overrightarrow{L_{1}^{1}} \times \overrightarrow{L_{2}^{1}}\right)}{\left(\overrightarrow{L_{1}^{1}} \times \overrightarrow{L_{2}^{1}}\right) \cdot\left(\overrightarrow{L_{1}^{1}} \times \overrightarrow{L_{2}^{1}}\right)}\right) * \overrightarrow{L_{1}^{1}} \\
L p_{2}^{1}=p_{2}^{1}+\left(\frac{\left(\overrightarrow{L_{1}^{1}} \times\left(p_{1}^{1}-p_{2}^{1}\right)\right) \cdot\left(\overrightarrow{L_{1}^{1}} \times \overrightarrow{L_{2}^{1}}\right)}{\left(\overrightarrow{L_{1}^{1}} \times \overrightarrow{L_{2}^{1}}\right) \cdot\left(\overrightarrow{L_{1}^{1}} \times \overrightarrow{L_{2}^{1}}\right)}\right) * \overrightarrow{L_{2}^{1}} \\
L p^{1}=\frac{L p_{1}^{1}+L p_{2}^{1}}{2} \\
E=\left|L p_{1}^{1}-L p_{2}^{1}\right|
\end{gathered}
$$

$L p^{1}$ is the $3 \mathrm{D}$ position of light 1 in the world coordinate system. $L p_{1}^{1}$ is the point on vector $\overrightarrow{L_{1}^{1}}$ closest to $\overrightarrow{L_{2}^{1}}, L p_{2}^{1}$ is the point on vector $\overrightarrow{L_{2}^{1}}$ closest to $\overrightarrow{L_{1}^{1}}, E$ is the distance between these two points - which can be used to measure the accuracy of the calculation. If $E$ is zero then both light vectors intersect. However, due to error in estimating the light vector, the position of the highlight or sphere centre $E$ is not always zero or close to zero. So we use a threshold to establish when the estimated light position is not accurate. In this case the sphere can be positioned in additional places to improve the accuracy.

To calculate the position of light using the above method we need the position of at least two highlights on the sphere surface. These highlights can be calculated by first calculating the centre of the sphere. As the actual size of the sphere, focal length of the camera and physical pixel size of camera 
world coordinate system.

$$
\begin{gathered}
c(X, Y, Z)=\left[\frac{-x}{f_{x}} Z, \frac{-y}{f_{y}} Z, Z\right] \\
Z=\frac{\text { focalLength } * \text { sphereActualRadius }}{\text { pixelLength } * \text { spherePixelRadius }}
\end{gathered}
$$

Where $Z$ is the distance of sphere centre from camera in the z direction, $f_{x}$ and $f_{y}$ are the focal length in pixels in $x$ and $y$ direction. Once the centre of sphere $c$ is known, the surface normal $\vec{n}$ at point $p$ (highlight pixel position) can be used to calculate $p$ from equation 15 .

$$
p(X, Y, Z)=c(X, Y, Z)+k * n(X, Y, Z)
$$

$k$ is a constant required to calculate $p$. As $p$ lies on the surface of the sphere $|p-c|$ should be equal to the sphere radius and by using value of $p$ from equation 15 we can solve the value of $k$ from the following equations.

$$
|c+k \vec{n}-c|=\text { sphereActualRadius }
$$

$$
|\vec{n}|=1
$$

$$
k=\text { sphereActualRadius }
$$

175 Once the value of $k$ is calculated, it can be used in equation 15 to calculate the position of the highlight on the sphere surface in real world coordinates; as shown in equation 19. 


$$
p(X, Y, Z)=c(X, Y, Z)+\text { sphereActualRadius } * \vec{n}
$$

\subsection{Object Distance Estimation}

The object distance from the camera is calculated by using the Diffused Maxima Region (DMR), which is calculated by taking the absolute of the dot product between the pseudo light vector and pseudo surface normal, and then applying a threshold; as shown in equation 20. During experimentation we have found that for most cases the threshold is greater then or equal to 0.9 .

$$
D M R_{i}=\left|\vec{N} \cdot \overrightarrow{L^{i}}\right|>0.9
$$

$\overrightarrow{L^{i}}$ is a pseudo light vector for light $\mathrm{i}$ and $\vec{N}$ is the pseudo surface normal at each pixel. The pseudo light vector $\overrightarrow{L^{i}}$ is calculated during the calibration process by placing the sphere at the centre of the field of view, it is assumed to be same for every pixel. The centre of the $D M R$ gives us the point where the surface normal and the light vector are approximately aligned. Many $\operatorname{DMR}(s)$ can exist on the surface of an object but the region with maximum pixel area is considered to be the best choice. Lights are arranged in a square arrangement as shown in Figure 4(a) and the dot product of the light vectors with surface normals are shown in Figure 2. Higher value of dot product means it is more close to diffused maxima. Figure 2 shows the four selected $D M R$ centres plotted on a height map of a synthetic sphere and a real human dummy torso. 


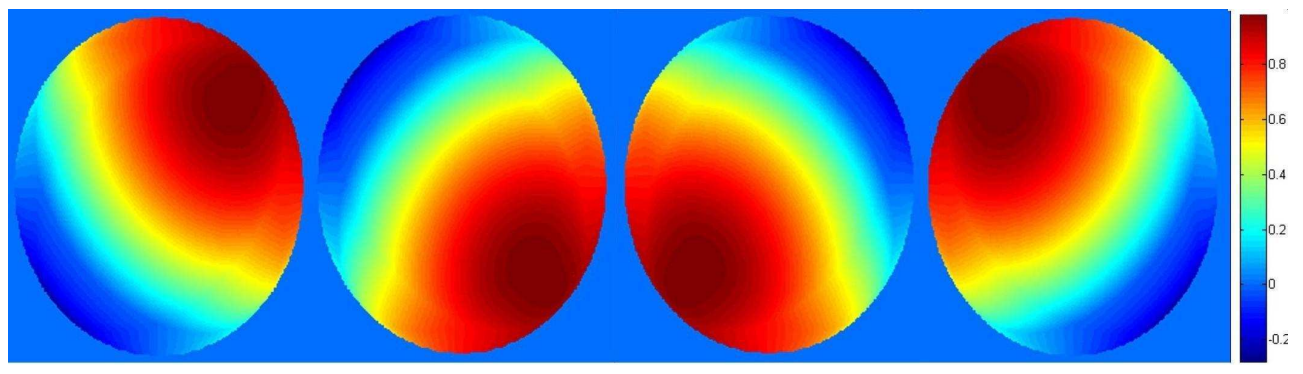

(a)

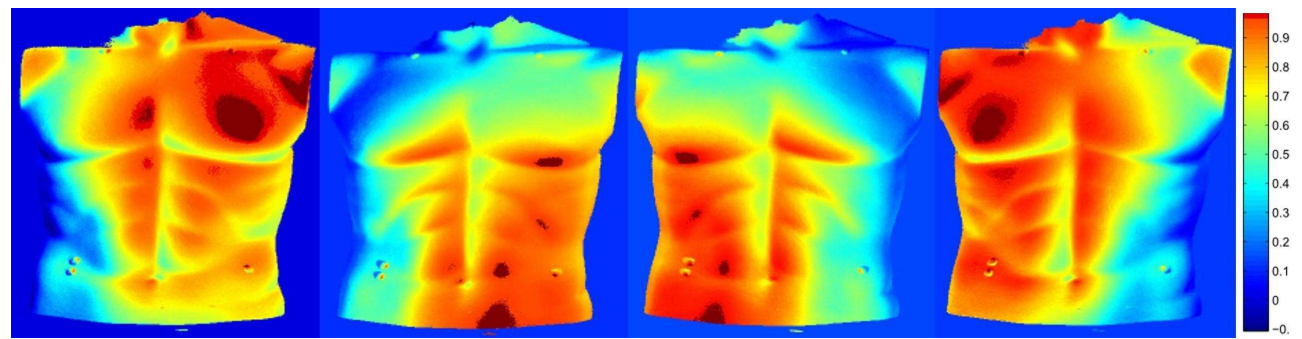

(b)

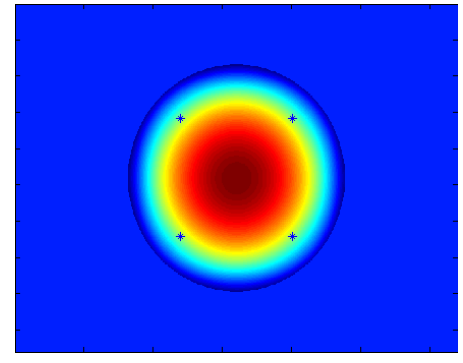

(c)

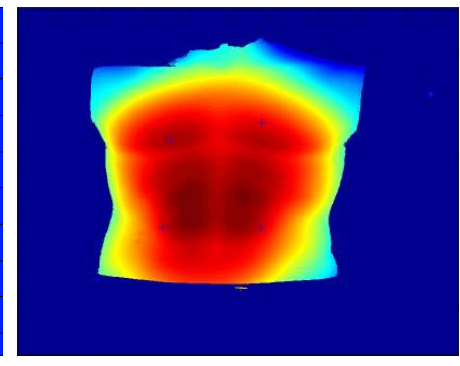

(d)

Figure 2: (a) and (b) dot product of image with its light vectors. Diffused maxima regions are in highlighted in dark red colour. (c) and (d) Diffused Maxima Regions centres are plotted on Height Map 


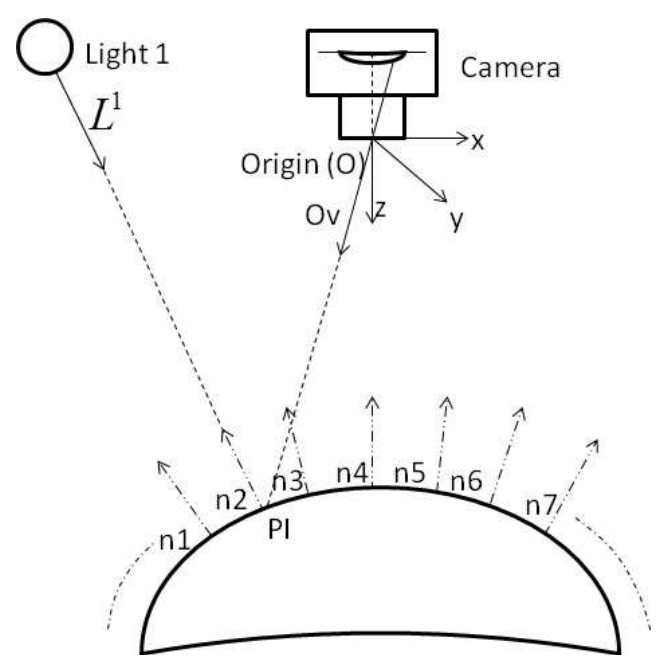

(a)

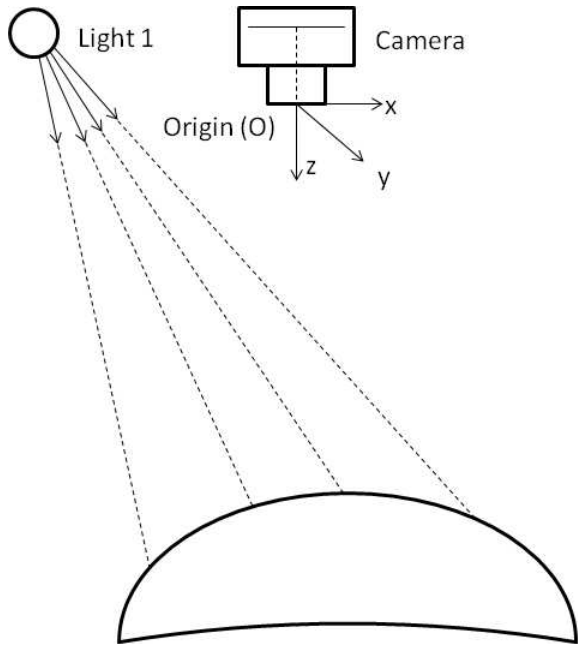

(b)

Figure 3: (a) Depth calculation using DMR and intersection of vector $O v$ and $L^{1}$. (b) Light vector calculation on each point of object surface.

Once the $D M R$ centre is identified in the image plane, a vector $\overrightarrow{O v}$ can be created from the $D M R$ centre to the centre of the lens $O$, as shown in Figure 3(a). $O$ is also the origin of the world coordinate system. Now by using origin $O$, position of light $L P$, light vector $\overrightarrow{L^{1}}$ and vector $\overrightarrow{O v}$, we can determine the intersection point of these two vectors in world coordinates by using equations 9,10 and 11 . The average of the $Z$ coordinate of these points of intersection is the estimated distance of the object from the camera.

\subsection{Per pixel light direction calculation}

Once the distance of the object is known from the camera, an imaginary plane parallel to the image plane is created. The pseudo height of the object is then defined relative to this plane by adding the reconstructed surface from pseudo normals; so that new light vectors for each pixel point for each 
light are created as shown in Figure 3(b). The pseudo height of the object is calculated by integrating [29] the pseudo surface normal $\mathrm{N}$ and then scaling the height to compensate for the camera distance.

Traditional photometric stereo assumes that the light direction is same across the whole scene but in reality, particularly where the object has a comparable size to the illumination working distance, it is clear that this varies; as shown in Figure 3(b). This variation needs to be considered for accurate surface normal calculation because any variations in the illumination position are finally interpreted as uncertainty in recovered surface normals. For our synthetic imaging setup Table 2 shows the range of light vectors in terms of tilt and slant of a plane at a known distance from the camera, compared to traditional photometric stereo where the tilt and slant angle of illumination are normally assumed fixed.

Table 2: Tilt and Slant Light angle range for traditional PS and proposed method

\begin{tabular}{|l|l|l|l|l|}
\hline \multirow{2}{*}{} & \multicolumn{2}{|c|}{ Our Method } & \multicolumn{2}{c|}{ Traditional PS } \\
\cline { 2 - 5 } & $\begin{array}{l}\text { Max/Min } \\
\text { Tilt(degree) }\end{array}$ & $\begin{array}{l}\text { Max/Min } \\
\text { Slant(degree) }\end{array}$ & $\begin{array}{l}\text { Tilt (de- } \\
\text { gree) }\end{array}$ & $\begin{array}{l}\text { Slant (de- } \\
\text { gree) }\end{array}$ \\
\hline Light 1 & $-8.8 /-76.2$ & $83.3 / 57$ & -45 & 70.5 \\
\hline Light 2 & $171.2 / 103.8$ & $83.4 / 57.2$ & 135 & 70.5 \\
\hline Light 3 & $-103 /-171$ & $83 / 57$ & -135 & 70.5 \\
\hline Light 4 & $70.5 / 8.8$ & $83 / 57$ & 45 & 70.5 \\
\hline
\end{tabular}




\section{Experiments and Results}

Experiments were performed on range of synthetic images as well as with real images. For real images a setup based on a Teledyne DALSA Genie HM1400 1.4 Mega pixel monochrome camera and High power LEDs was designed as shown in Figure 4(a). A commercial 3dMD [30] system is used to acquire ground truth data as this system has a reported $0.2 \mathrm{~mm}$ accuracy in depth measurement.

Figure 5(a) shows the error $(\mathrm{mm})$ in the calculation of object distance from camera when the initial calibration (pseudo light vectors) of the setup is performed with the specular sphere located approximately at $2000 \mathrm{~mm}$ from the camera. The $\sim \pm 20 \mathrm{~mm}$ uncertainty is found when the object is moved from $1800 \mathrm{~mm}$ to $2200 \mathrm{~mm}$ from the camera. This is relatively high compared to other 3D range finding technologies, however the system can achieve a recovery in pixel level which is not provided by any other 3D imaging systems.

To test the accuracy of the surface normals acquired from the proposed method we have used Mean Angular Error (MAE) as the measure of accuracy. MAE is calculated by taking the cosine inverse of the dot product of a ground truth surface normal and a calculated surface normal. Table 3 summaries the Mean Angular error calculated from a synthetic as well as real images. Table 3 shows that the mean error in the height calculation of the reconstructed surface is improved around 2-6 $\mathrm{mm}$ in height and there is around 2-3 degree improvement in surface normal estimation.

Figure 5(b) shows mean angular error (degree) when the object is moved from $1900 \mathrm{~mm}$ to $2100 \mathrm{~mm}$ with the initial light vector for traditional photometric stereo and a pseudo light vector for our proposed method calculated 


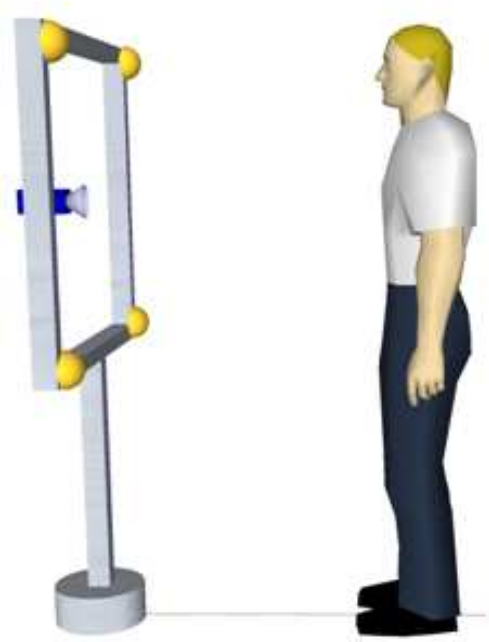

(a)

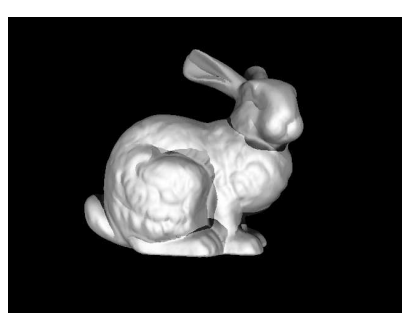

(b)

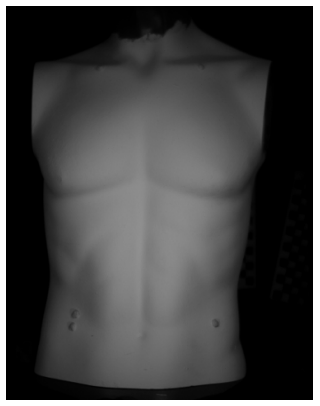

(d)

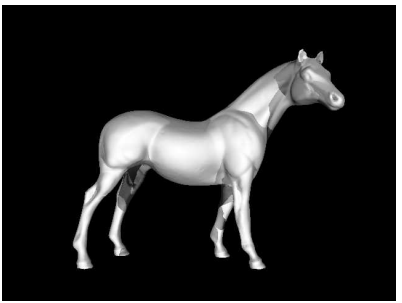

(c)

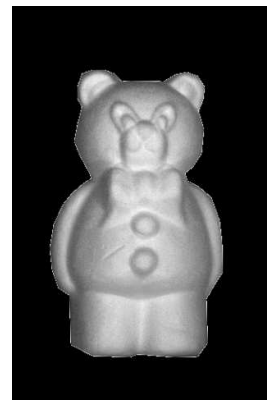

(e)

(f)

Figure 4: (a) Image acquisition Setup. (b-f) images of Objects used in Experiments 


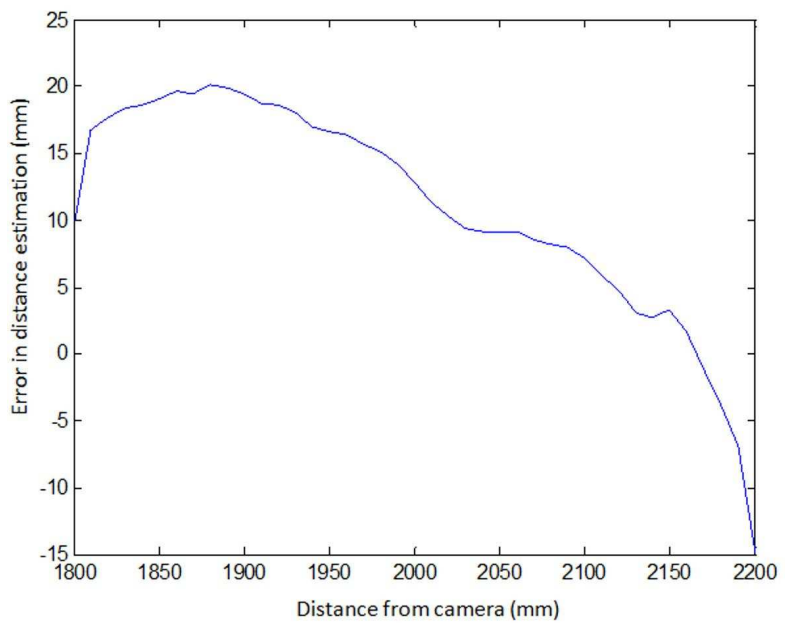

(a)

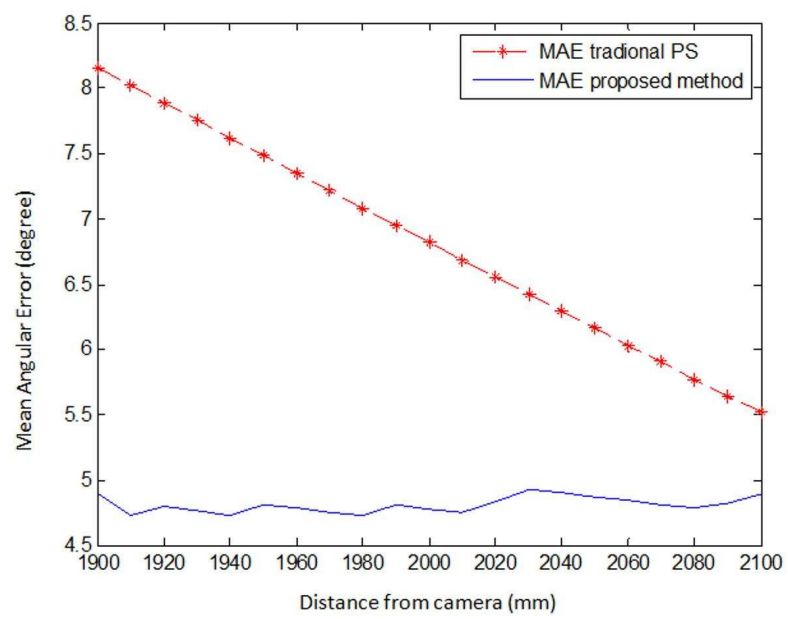

(b)

Figure 5: (a) Absolute Error in distance estimation from camera to object. (b) Mean angular error in surface normals. 

position while the proposed method has a constant low MAE. is highly dependent on the location of object with respect to the calibration

Table 3: Mean Error

\begin{tabular}{|l|l|l|l|l|}
\hline & \multicolumn{3}{|l|}{$\begin{array}{l}\text { Mean Angular Error in } \\
\text { surface normal(degree) }\end{array}$} & $\begin{array}{l}\text { Mean Height Er- } \\
\text { ror(mm) }\end{array}$ \\
\cline { 2 - 5 } & $\begin{array}{l}\text { Traditional } \\
\text { PS [3] }\end{array}$ & $\begin{array}{l}\text { Our } \\
\text { Method }\end{array}$ & $\begin{array}{l}\text { Traditional } \\
\text { PS [3] }\end{array}$ & $\begin{array}{l}\text { Our } \\
\text { Method }\end{array}$ \\
\hline Synthetic Sphere & 6.53 & 4.53 & 14.586 & 9.108 \\
\hline Synthetic Bunny & 3.95 & 2.36 & 15.378 & 11.826 \\
\hline Synthetic Horse & 3.90 & 2.33 & 9.174 & 6.608 \\
\hline Polystyrene Sphere & 6.72 & 4.61 & 15.642 & 10.714 \\
\hline Human Dummy & 6.88 & 4.86 & 17.006 & 11.066 \\
\hline Polystyrene Face & 7.1 & 5.2 & 15.642 & 13.530 \\
\hline Polystyrene Owl & 7.5 & 4.18 & 15.224 & 11.044 \\
\hline
\end{tabular}

at $2000 \mathrm{~mm}$. It can be found that the MAE in traditional photometric stereo 
photometric stereo imaging setups. In comparison, Figure 6(b) is more flat and closer to the ground truth. This is because the geometric distortion is partially removed by considering the lighting distance from the object surface. The same phenomena can be observed clearly by plotting slices of the surfaces as shown in Figure 7.

Figure 7 shows slices of reconstructed surfaces. When comparing proposed method (which estimates distance of object from the camera and calculates light vector for every pixel using distance estimation), with traditional photometric stereo (which assumes the same lighting direction for each pixel), it is clear that the proposed method calculates more accurate surface normals and hence better surface reconstruction.

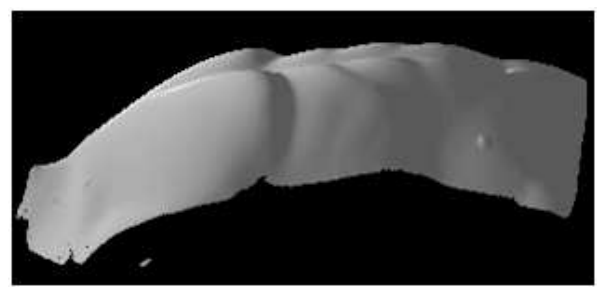

(a)

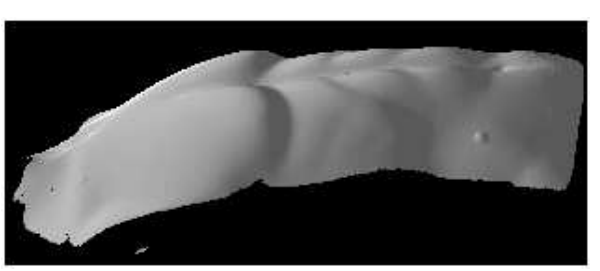

(b)

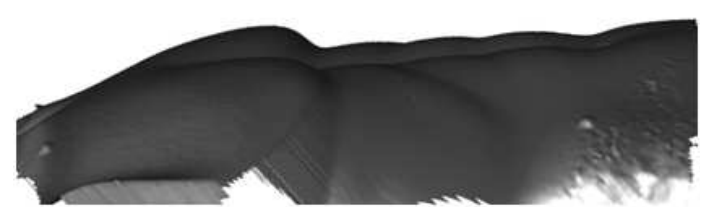

(c)

Figure 6: (a) Integrated surface using traditional PS. (b) Integrated surface using Proposed method. (c) Surface scanned from 3dMD as a ground truth. 


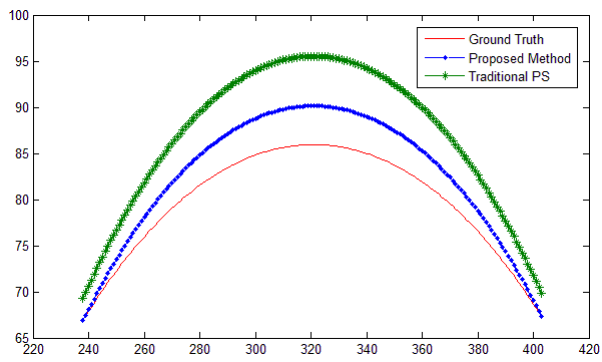

(a) Synthetic Sphere

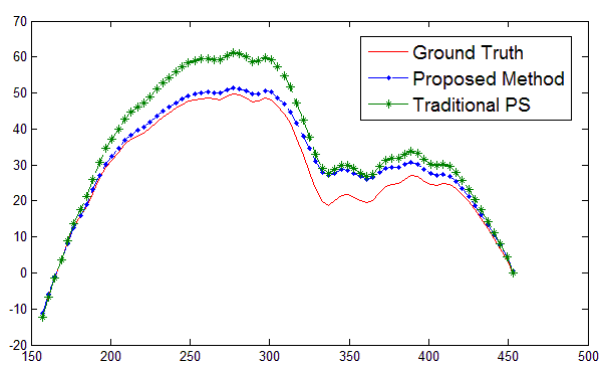

(c) Synthetic Bunny

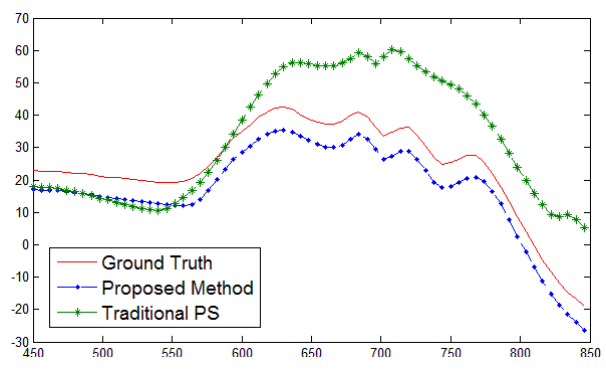

(e) Polystyrene Face

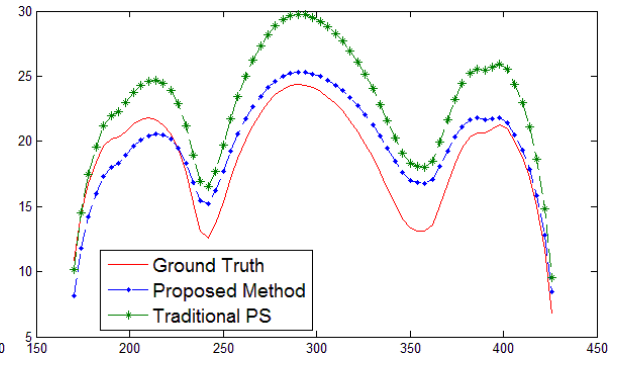

(b) Synthetic Horse

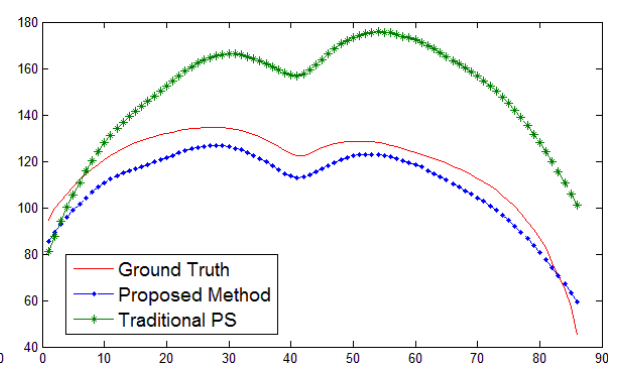

(d) Human Torso Dummy

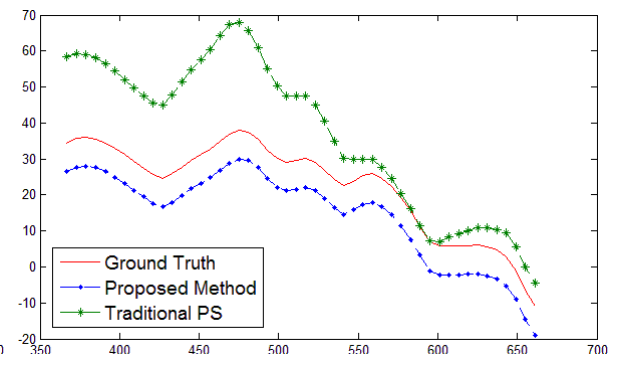

(f) Polystyrene Owl

Figure 7: Slices of Integrated Surface

\section{Conclusion}

This approach offers a useful way to add range data, improving accuracy and reducing distortion in PS acquired reconstructed 3D surfaces. Distortion in PS derived 3D surface data is a well know limitations of the method and 
its solution offers opportunity for taking advantage of the PS methodology in a new range of challenging applications, including accurate real-time reconstruction non-rigid 3D surfaces, such as the moving human chest. In this paper we presented a new method to calculate light vectors dynamically for improving photometric stereo 3D surface reconstruction performance. The improvement in light vector estimation is achieved through calculating the distance of the object from the camera using diffused maxima region and then using this distance to calculate per-pixel light vector dynamically. This dynamic calculation can be done in real-time (i.e. real-time reconstruction of a deforming 3D shape, such as a human chest). By using the proposed method the error in surface normal estimation is reduced to become almost constant and independent from the working distance. Experiments performed on synthetic and real scenes shows there is improvement of up to $45 \%$ in surface normal and up to $6 \mathrm{~mm}$ in the reconstructed surface height.

\section{References}

[1] S. Barsky, M. Petrou, The 4-source photometric stereo technique for three-dimensional surfaces in the presence of highlights and shadows, IEEE Transactions on Pattern Analysis and Machine Intelligence 25 (10) (2003) 1239-1252. doi:10.1109/TPAMI.2003.1233898.

[2] J. Sun, M. Smith, L. Smith, S. Midha, J. Bamber, Object surface recovery using a multi-light photometric stereo technique for non-Lambertian surfaces subject to shadows and specularities, Image and Vision Computing 25 (7) (2007) 1050-1057. doi:10.1016/j.imavis.2006.04.025. 
[3] R. Woodham, Photometric method for determining surface orientation from multiple images, Optical engineering 19 (1) (1980) 139-144.

[4] T. Higo, Y. Matsushita, N. Joshi, K. Ikeuchi, A hand-held photometric stereo camera for 3-D modeling, IEEE 12th International Conference on Computer Vision (2009) 1234-1241doi:10.1109/ICCV.2009.5459331.

[5] C. Hern, G. Vogiatzis, C. Hernández, Self-calibrating a real-time monocular 3d facial capture system, International Symposium on 3D Data Processing, Visualization and Transmission (3DPVT).

[6] A. Jones, G. Fyffe, X. Yu, W.-C. C. Ma, J. Busch, R. Ichikari, M. Bolas, P. Debevec, Head-Mounted Photometric Stereo for Performance Capture, Conference for Visual Media Production (2011) 158164doi:10.1109/CVMP.2011.24.

[7] J. Ahmad, J. Sun, L. Smith, M. Smith, J. HENDERSON, A. MAJUMDAR, Novel Photometric Stereo Based Pulmonary Function Testing, in: 3rd internation conference and exhibition on 3D Body Scanning Technologies, Lugano, Switzerland., 2012.

[8] J. Sun, M. Smith, L. Smith, L. Coutts, R. Dabis, C. Harland, J. Bamber, Reflectance of human skin using colour photometric stereo: with particular application to pigmented lesion analysis., Skin research and technology 14 (2) (2008) 173-9. doi:10.1111/j.1600-0846.2007.00274.x.

[9] M. F. Hansen, G. A. Atkinson, L. N. Smith, M. L. Smith, 3D face reconstructions from photometric stereo using near infrared and visible 
light, Computer Vision and Image Understanding 114 (8) (2010) 942951. doi:10.1016/j.cviu.2010.03.001.

[10] J. Sun, M. Smith, L. Smith, A. Farooq, Examining the uncertainty of the recovered surface normal in three light photometric stereo, Image and Vision Computing 25 (7) (2007) 1073-1079.

[11] M. Kobayashi, T. Okabe, Y. Matsushita, Y. Sato, Surface Reconstruction in Photometric Stereo with Calibration Error, in: International Conference on 3D Imaging, Modeling, Processing, Visualization and Transmission, Inst. of Ind. Sci., Univ. of Tokyo, Tokyo, Japan, IEEE, Hangzhou, China, 2011, pp. 25-32. doi:10.1109/3DIMPVT.2011.13.

[12] I. Horovitz, N. Kiryati, Depth from gradient fields and control points: Bias correction in photometric stereo, Image and Vision Computing 22 (9) (2004) 681-694. doi:10.1016/j.imavis.2004.01.005.

[13] D. Junyu, G. McGunnigle, S. Liyuan, F. Yanxia, W. Yuliang, Improving photometric stereo with laser sectioning, 12th IEEE International Conference on Computer Vision Workshops (ICCV Workshops) (2009) 1748-1754doi:10.1109/ICCVW.2009.5457494.

[14] C. Hernández Esteban, G. Vogiatzis, R. Cipolla, C. Hernández, Multiview photometric stereo., IEEE transactions on pattern analysis and machine intelligence 30 (3) (2008) 548-54. doi:10.1109/TPAMI.2007.70820.

[15] H. Du, D. Goldman, S. Seitz, Binocular Photometric Stereo, in: British Machine Vision Conference 2011, British Machine Vision Association, 2011, pp. 84.1-84.11. doi:10.5244/C.25.84. 
16] W. de Boer, J. Lasenby, J. Cameron, R. Wareham, S. Ahmad, C. Roach, W. Hills, R. Iles, SLP: A Zero-Contact Non-Invasive Method for Pulmonary Function Testing, British Machine Vision Conference 2010 (2010) 85.1-85.12doi:10.5244/C.24.85.

[17] C. Wu, Y. Liu, Q. Dai, B. Wilburn, Fusing multiview and photometric stereo for $3 \mathrm{D}$ reconstruction under uncalibrated illumination., IEEE transactions on visualization and computer graphics 17 (8) (2011) 108295. doi:10.1109/TVCG.2010.224.

[18] P. Favaro, T. Papadhimitri, A closed-form solution to uncalibrated photometric stereo via diffuse maxima, in: IEEE Conference on Computer Vision and Pattern Recognition, IEEE, 2012, pp. 821-828. doi:10.1109/CVPR.2012.6247754.

[19] P. N. Belhumeur, D. J. Kriegman, A. L. Yuille, The Bas-Relief Ambiguity, International Journal of Computer Vision 35 (1) (1999) 33-44. doi:10.1023/A:1008154927611.

[20] L. Smith, M. Smith, The virtual point light source model the practical realisation of photometric stereo for dynamic surface inspection, in: Proceedings of the 13th international conference on Image Analysis and Processing, ICIAP'05, Springer-Verlag, Berlin, Heidelberg, 2005, pp. 495-502. doi:10.1007/11553595_61.

[21] I. Ashdown, Near-field photometry: Measuring and modeling complex 3-d light sources, ACM SIGGRAPH95 Course Notes-Realistic Input for Realistic Images. 
[22] B. Kim, P. Burger, Depth and shape from shading using the photometric stereo method, CVGIP: Image Understanding 54 (3) (1991) 416-427.

[23] Y. Iwahori, H. Sugie, N. Ishii, Reconstructing shape from shading images under point light source illumination, in: Pattern Recognition, 1990. Proceedings., 10th International Conference on, Vol. i, 1990, pp. 83 -87 vol.1. doi:10.1109/ICPR.1990.118069.

[24] J. J. Clark, Active photometric stereo, in: Computer Vision and Pattern Recognition, 1992. Proceedings CVPR '92., 1992 IEEE Computer Society Conference on, 1992, pp. 29-34. doi:10.1109/CVPR.1992.223231.

[25] R. Kozera, L. Noakes, Noise reduction in photometric stereo with nondistant light sources, Computer Vision and Graphics (2006) 103-110.

[26] M. L. Smith, L. N. Smith, Dynamic photometric stereo-a new technique for moving surface analysis, Image Vision Comput. 23 (9) (2005) 841852. doi:10.1016/j.imavis.2005.01.007.

[27] A. Varnavas, V. Argyriou, J. Ng, A. A. Bharath, Dense photometric stereo reconstruction on many core GPUs, 2010 IEEE Computer Society Conference on Computer Vision and Pattern Recognition - Workshops (2010) 59-65doi:10.1109/CVPRW.2010.5543152.

[28] F. Dunn, I. Parberry, 3D math primer for graphics and game development, Wordware Publishing, Inc, 2011.

[29] T. Simchony, R. Chellappa, M. Shao, Direct analytical methods for solving Poisson equations in computer vision problems, IEEE Transactions 
on Pattern Analysis and Machine Intelligence 12 (5) (1990) 435-446.

388 doi:10.1109/34.55103.

389 [30] 3dMD, www.3dmd.com/3dmdface.html. 